\title{
Spatio-temporal patterns of pre-eclampsia and eclampsia in relation to drinking water salinity at the district level in Bangladesh from 2016 to 2018
}

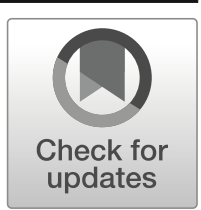

\author{
Jessie Pinchoff ${ }^{1}$ (D) Mohammad Shamsudduha $^{2}$. \\ Sharif Mohammed Ismail Hossain ${ }^{3}$. Abdullah Al Mahmud Shohag ${ }^{3}$. \\ Charlotte E. Warren ${ }^{4}$
}

Published online: 25 November 2019

C) The Author(s) 2019

\begin{abstract}
This analysis examines whether salinity in drinking water is associated with preeclampsia and eclampsia (PE/E), a leading cause of maternal morbidity and mortality. Bangladesh's national health information system data were extracted at the district level ( $n=64)$ to assess PE/E rates, and these were overlaid with three environmental measures approximating drinking water salinity, remotely sensed low-elevation coastal zone (LECZ), monthly rainfall data, and electrical conductivity of groundwater (i.e., water salinity). Results from a negative binomial fixed effects model suggest $\mathrm{PE} / \mathrm{E}$ rates are higher with less rainfall (dry season), lower population density, and that district level rates of PE/E increase with higher groundwater salinity and in the high risk LECZ category closest to the coast. Results suggest that drinking water salinity may be associated with $\mathrm{PE} / \mathrm{E}$ and that using national health surveillance data can improve understanding of this association. This approach can potentially be leveraged in the future to inform targeted interventions to high risk regions and times.
\end{abstract}

Keywords Remote sensing $\cdot$ Bangladesh $\cdot$ Eclampsia $\cdot$ Salinity $\cdot$ Sea level rise $\cdot$ Coastal populations

Jessie Pinchoff

jpinchoff@popcouncil.org

1 Population Council-NY, One Dag Hammarskjold Plaza \#3, New York, NY 10017, USA

2 Department of Geography, University of Sussex, Arts C C308, Brighton BN1 9SJ, UK

3 Population Council-Bangladesh, House \#12 Road \#25/30, Gulshan-1, Dhaka 1212, Bangladesh

4 Population Council-DC, 4301 Connecticut Avenue NW Suite 280, Washington, DC 20008, USA 


\section{Introduction}

Hypertensive disorders in pregnancy (HDPs) are major causes of severe morbidity, long-term disability and mortality of both women and their newborn babies. Globally, about $14 \%$ of all maternal deaths are due to HDPs, of which pre-eclampsia and eclampsia (PE/E) are associated with the majority of morbidity and mortality (Say et al. 2014). The precise medical cause of eclampsia is unknown, and definitions vary. Generally, pre-eclampsia is diagnosed when a woman experiences a rapid elevation in blood pressure (hypertension) and increased levels of protein in the urine after 20 weeks of gestation (significant proteinuria $>0.3 \mathrm{~g} /$ day or $>30 \mathrm{mg} / \mathrm{mmol}$ of urinary creatinine in a random sample); if untreated, this can lead to eclampsia, a disorder characterized by seizures and other complications that can ultimately lead to the death of the woman and/or her fetus (WHO 2011, Kirk and Dempsey 2016, Magee et al. 2016). Early diagnosis and management are critical for improving maternal and newborn outcomes. PE/E can be detected through routine screening of blood pressure and urine protein levels during antenatal care visits, and low dose aspirin and calcium can be used prophylactically (Redman 2011, Mone and MCAuliffe 2014, Kirk and Dempsey 2016). A pregnant woman with high blood pressure can be prescribed antihypertensive medications safe for use during pregnancy, and eclamptic convulsions can be prevented and controlled with a dose of injectable magnesium sulfate $\left(\mathrm{MgSO}_{4}\right)(\mathrm{ACOG}$ 2013). Despite the highly effective, low cost, and relatively simple prevention methods available, PE/E persists as a leading cause of death among pregnant women globally. While preventable, morbidity and mortality persist due in part to shortages of $\mathrm{MgSO}_{4}$ and lack of trained facility staff able to administer it. The mechanisms for PE/E are not yet well understood, so the literature continues to explore its risk factors, in order to better understand the condition and develop programs and policy to reduce its prevalence. Researchers have identified several risk factors that may be associated with $\mathrm{PE} / \mathrm{E}$, including health and lifestyle risk factors, such as smoking, alcohol consumption, obesity, family history, nulliparity, and chronic hypertension (Duckitt and Harrington 2005, UN General Assembly 2011). Several micronutrient, mineral, and trace element deficiencies are also reportedly linked with PE/E risk; a recent systematic review identified associations between iron deficiency, low magnesium, and selenium during pregnancy with $\mathrm{PE} / \mathrm{E}$ (Elmugabil et al. 2016, Kirk and Dempsey 2016, Xu et al. 2016). An association between hypertension and PE/E specifically, with high salinity (salt) drinking water, has been reported, prompting this research study.

There is sufficient epidemiological evidence associating high salt intake with risk of hypertension in children and adults (He and Macgregor 2009, EPA 2014). A Cochrane review meta-analysis of 34 randomized trials found a mean reduction in urinary sodium levels caused a significant reduction in systolic and diastolic blood pressure, and that recommendations to reduce dietary salt intake would result in lower blood pressure (He and Macgregor 2009). Salt can be consumed through food but also through water. The World Health Organization (WHO) has prioritized research regarding the adverse health impacts of consumption of highly saline water (McMichael 2003). According to a 2005 Cochrane review, research on salt intake and hypertension specifically during pregnancy and the link with PE/E is less clear (Duley et al. 2005). Research suggesting salt in drinking water may be associated with higher risk of $\mathrm{PE} / \mathrm{E}$ has been reported almost exclusively in Bangladesh. This is due in large part to Bangladesh's low-lying 
elevation and coastal proximity, placing it at high risk for saline intrusion from sea level rise and coastal inundation. About $98 \%$ of the population drinks from tube wells that access groundwater, making this a potentially serious and widespread risk factor for hypertension and PE/E in Bangladesh, as well as other countries with similar geographic features.

\section{Research objectives}

This study aims to measure the association between district level PE/E rates and drinking water salinity (as approximated by low-elevation coastal zone (LECZ) and groundwater salinity) by season (as defined by monthly rainfall) across Bangladesh between 2016 and 2018. It is the first study to explore this association at the national level. We used geographic location to link various datasets, accessing variables that are not reported together otherwise. This paper describes the relationship between rates of $\mathrm{PE} / \mathrm{E}$ and salinity using an approach that could be replicated in the future to systematically and routinely link health surveillance data with environmental or remotely sensed data. If standardized indicators are used, this methodology could allow for comparisons across countries and environmental settings where sea level rise and salinity may be risk factors for health outcomes such as PE/E. This study describes $\mathrm{PE} / \mathrm{E}$ in Bangladesh and our approach to detect spatial and temporal variation in district level $\mathrm{PE} / \mathrm{E}$ rates using secondary analysis.

\section{Coastal Bangladesh and PE/E}

Bangladesh reports $\mathrm{PE} / \mathrm{E}$ as the second leading direct cause of obstetric deaths nationally, accounting for 24\% of maternal deaths (National Institute of Population Research and Training (NIPORT) 2017). A short report in 2008 detected an elevated rate of hypertensive disorders in pregnant women in a health facility in Dacope, a coastal area in Bangladesh (Khan et al. 2008). Due to the concurrent trends in sea level rise and reported contamination of fresh drinking water sources with sea water due to extreme weather events, a link with drinking water salinity was hypothesized. Local doctors and members of the community identified increased salinity as the cause, but no epidemiological research was available at that time (Khan et al. 2008). The authors followed up with a descriptive study in 2011 that detected a link between drinking water salinity, urine sodium levels, and blood pressure among a small sample of women in Dacope, the same coastal region of Bangladesh (Khan et al. 2011). They also detected a seasonal trend, with higher rates of hypertension reported in the dry season. In 2014, this association was again confirmed in a case-control study that measured sodium levels in household drinking water sources and found that women consuming tube well (groundwater) were at higher disease risk than those drinking rainwater; a dose response relationship between salinity and both pre-eclampsia and gestational hypertension was reported (Khan et al. 2014). Since then, three studies have also linked drinking water salinity and blood pressure among a small sample of the general population (Scheelbeek et al. 2016), among young adults (Talukder et al. 2016), and a survey of the general population through the Assessing Health, Livelihoods, 
Ecosystem Services and Poverty Alleviation in Populous Deltas (ESPA Deltas) project (Nahian et al. 2018). Another study detected a link between drinking water salinity and infant mortality using Demographic and Health Survey (DHS) data (Dasgupta et al. 2015).

In Bangladesh, salinity has already begun to increase in soil and water and is projected to increase further (Khan et al. 2014). Two-thirds of Bangladesh's land area is less than $5 \mathrm{~m}$ above sea-level (Dasgupta et al. 2015). The LECZ is vulnerable to coastal threats including progressive inundation from sea level rise, heightened storm damage, loss of wetlands, and increased salinity from saltwater intrusion (Dasgupta et al. 2015). Although groundwater is abundant in coastal Bangladesh, saline water is thought to intrude into the aquifer system due to reductions of upstream freshwater flow and exacerbation by shrimp farming and over abstraction of groundwater for irrigation (Hasan et al. 2013). Bangladesh's population, mainly in rural areas, relies heavily on natural water sources such as ponds, rivers, and tube wells for drinking water. Groundwater typically contains higher sodium levels than surface water. A recent study found mean sodium concentrations in drinking water in coastal Bangladesh of $700 \mathrm{mg} / \mathrm{L}$, in some areas exceeding $1500 \mathrm{mg} / \mathrm{L}$; the WHO recommends a maximum sodium intake of $2000 \mathrm{mg}$ that can be easily exceeded in this region between dietary salt intake and drinking water (Scheelbeek et al. 2016). Approximately 20 million people live in coastal areas of Bangladesh affected by varying degrees of salinity in drinking water (MOEF 2006). If drinking saline water leads to high blood pressure, the coastal population of Bangladesh is at significant risk of adverse health outcomes including $\mathrm{PE} / \mathrm{E}$. This threat is projected to increase unless alternative drinking water sources can be identified. Further research is necessary to better understand the link between drinking water salinity and $\mathrm{PE} / \mathrm{E}$ and if possible, to monitor the problem. While the studies cited above are critical for elucidating the links between drinking water salinity and $\mathrm{PE} / \mathrm{E}$, all are based on intensive data collections that do not allow for routine monitoring or national level assessment. Methods that use freely available satellite data and health surveillance data may allow for routine monitoring and identification of high risk 'hotspots' that can add to the epidemiological evidence and better target future public health programs and interventions.

\section{Opportunities for surveillance data}

All except one of the studies described above were conducted with very small sample sizes and only in coastal areas of Bangladesh (the exception used DHS data that does not record PE/E). Implementing detailed surveys with anthropometric data such as blood pressure and household visits to test water samples can be costly and time intensive. We aimed to detect the relationship between drinking water salinity and $\mathrm{PE} / \mathrm{E}$ using routinely collected health surveillance data reported in the District Health Information System (DHIS2). DHIS2 is a software platform developed to manage health information systems (HIS); it is currently used in 60 low- and middle-income countries globally, including Bangladesh. DHIS2 allows facilities and administrative areas to report health indicators allowing for collection and aggregation of health data, including cases of $\mathrm{PE} / \mathrm{E}$. Although there are certain valid concerns regarding the quality of DHIS2 data, overall, it presents an opportunity for analysis of routinely collected 
health surveillance data (Dehnavieh et al. 2019). Routinely collected data from health facilities is relatively inexpensive but relies on people and institutions to enter data and ensure its completeness, consistency, and timeliness. Although not perfect, the quality of data in DHIS2 is rapidly improving with the objective of making it a reliable, representative source of health information. DHIS2 is continually rolling out new built in data quality tools and checks to address data quality (Haugen et al. 2017). Benefits of these systems include the potential real-time analysis of health data and the opportunity to link with various environmental datasets using location information.

Health surveillance data can be utilized in many ways, including to document or track progress towards public health goals, monitor the epidemiology and magnitude of a certain disease, serve as an early warning system to detect impending public health emergencies, and potentially to inform the targeting of interventions (Dehnavieh et al. 2019). Health surveillance data and remotely sensed data could be effective tools to explore health and environment associations, such as between salinity and PE/E, especially if they can be routinely linked to allow for real-time detection of hotspots to inform program targeting. This also allows for an exploration of $\mathrm{PE} / \mathrm{E}$ and drinking water salinity for the entire country instead of just a coastal district, since the data are available throughout. This methodology can also allow for replication in other countries where DHIS2 data is collected, since rainfall and elevation are available globally. With the expansion in availability of satellite-derived data, the increasing accuracy of these data, and the diffusion of mobile data collection through platforms such as DHIS2, complex relationships between environment and health can be visualized and analyzed, to inform on the ground research that is necessary to confirm and expand upon the detected relationships. The use of publicly available satellite derived data and DHIS2 health surveillance data presents a new approach to exploring and describing the relationship between drinking water salinity and PE/E outcomes that has not been used before.

\section{Data and methods}

We overlaid data from multiple sources using their spatial location and aggregated the values to the district level for analysis. For this analysis, we used health surveillance data and multiple sources of environmental data including satellite derived LECZ and EC measured in groundwater surveys. No data was collected for this research; this is a secondary analysis of health surveillance, environmental, and climate datasets.

\section{Health surveillance data}

District level monthly health surveillance data reported between 2016 and 2018 were extracted from the national DHIS2 platform for Bangladesh. Facilities and regional offices upload monitoring data on various health outcomes related to pregnancy, maternal, and child health indicators that are aggregated to different administrative units, including the district level. To explore $\mathrm{PE} / \mathrm{E}$ rates as well as account for variation in total delivery volume per district, the number of PE/E cases and total facility-based deliveries were all aggregated to the district level ( $n=64$ districts). Platforms such as DHIS2 allow national health systems to collect, report, and analyze on a standardized 
set of indicators to understand trends and regional variation in health outcomes. The facilities report total $\mathrm{PE} / \mathrm{E}$ cases as well as total deliveries per month, aggregated to the district level. This was used as our outcome variable.

An additional variable for population density was explored, in part as a proxy for access to health services. This was calculated using population per district reported in 2011 divided by the area of each district in kilometers. The district population density was categorized into quartiles from lowest to highest density.

\section{Environmental data}

Three environmental data sources were overlaid with the district level DHIS2 health outcome data using R statistical software. The first is being located in the LECZ. The LECZ is defined as the contiguous and hydrologically connected zone of land along the coast and below $10 \mathrm{~m}$ elevation (McGranahan et al. 2007, Lichter et al. 2011). The LECZ bands of elevation per meter were categorized into two risk zones, $0-10 \mathrm{~m}$ (high risk) and $>10 \mathrm{~m}$ elevation (low/no risk). There can be slight misclassification in elevation, particularly for mangrove areas (Hawker et al. 2018). In the Sundarbans region of Bangladesh, this has been a challenge. However, a new elevation dataset called MERIT DEM is available that resolves these measurement issues and has less bias than previous digital elevation models such as the shuttle radar topography mission (SRTM); this allows for more accurate LECZ classification (Yamazaki et al. 2017, MacManus et al. 2019). This analysis uses the MERIT DEM dataset to classify LECZ. Being geographically located in the high risk LECZ category is predicted to increase risk of exposure to drinking water salinity due to sea level rise and coastal intrusion. The methods used to overlay spatial demographic data with LECZ exposure have been described previously (McGranahan et al. 2007). For each district, the elevation value of the majority of the pixels in that district was calculated (the mean value of the pixels in a district were also explored).

The second environmental dataset used is groundwater salinity at shallow depth $(<$ $150 \mathrm{~m}$ below ground level, bgl) at the national scale in Bangladesh. High levels of groundwater salinity are commonly recorded in coastal aquifers of southern Bangladesh. Groundwater salinity is generally defined by total dissolved solids (TDS) or electrical conductivity (EC); however, sometimes chemical constituents, such as chloride $(\mathrm{Cl})$, are also reported (Zahid et al. 2013, Shammi et al. 2019). Groundwater with greater concentration of dissolved ions has higher EC and hence higher salinity. In this study, groundwater salinity is expressed by its EC level, which has a unit of microSiemens per centimeter or $\mu \mathrm{S} / \mathrm{cm}$. This study applies district-level groundwater EC values, extracted from an interpolated, national-scale groundwater EC dataset based on 563 sample points collected from 2011 to 2013 (Shamsudduha, Joseph et al. 2019). There is currently no national, frequent monitoring of groundwater salinity in Bangladesh (Shamsudduha et al. 2019). Groundwater salinity does not necessarily follow coastal elevation; sea level rise influences surface water salinity (such as ponds, streams) and shallow groundwater (which is often accessed by tube wells), but deep groundwater may not be affected. In coastal Bangladesh, some tube wells are dug to over $200 \mathrm{~m}$ depth to access this deep groundwater, but freshwater is not always present even at depth and the installation of such deep tube wells is costly and thus not available for all coastal people (Lapworth et al. 2018). The mean, minimum, and 
maximum groundwater EC values per district were extracted. Mean groundwater EC was used in the final models. A binary variable was generated for safe drinking water based on the WHO cut off of $750 \mu \mathrm{S} / \mathrm{cm}$; above this level is considered unsafe.

\section{Climate data}

Neither LECZ nor the groundwater EC datasets are time varying; elevation is unlikely to significantly change over such a short time, and due to the intensive data collection required to measure and map groundwater EC, this was only collected at one time point for this nationally representative dataset (smaller area water quality assessments may be done more frequently). To account for the hypothesized seasonal variation in salinity in drinking water, monthly rainfall was extracted from the climate hazards infrared precipitation with stations (CHIRPS) dataset for all months 2016-2018 (Funk et al. 2015). We aggregated average rainfall per month and per district. The rainfall data was plotted over time to explore seasonal patterns between 2016 and 2018. It is hypothesized that salinity in surface water and shallow groundwater increases in the dry season due to evaporation as well as less water flow through river channels. The relationship is not straightforward due to the dynamic processes involved and paucity of data, but a recent study did find that saltwater intrusion was higher in the dry season compared with the wet season (Shammi et al. 2017). Average monthly rainfall at the district level was included in the final models to explore the hypothesized seasonal relationship.

\section{Statistical analysis}

EC, elevation, population density, and average rainfall pixel values were extracted and summarized at the district level. All variables were tabulated, and EC, elevation, and $\mathrm{PE} / \mathrm{E}$ rates per district per year were mapped to visualize any patterns. Monthly rainfall and proportion of total pregnancies with $\mathrm{PE} / \mathrm{E}$ were tabulated in a figure to explore the seasonal relationship between rainfall and $\mathrm{PE} / \mathrm{E}$ rates. The correlation between elevation and $\mathrm{EC}$ values were assessed and found to be positively associated. Rates of PE/E were our outcome variable of interest. LECZ, population density, EC, and rainfall were predictor variables. Year was a control variable.

$\mathrm{PE} / \mathrm{E}$ counts were plotted and observed to be heavily right skewed and overdispersed, making negative binomial models the best fit for the data (Cameron and Trivedi 2013). Compared with Poisson models, which are normally used for count data, negative binomial regression is preferred for modeling count variables when they are over-dispersed (i.e., the variance is greater than the mean). Poisson and negative binomial versions of the models were compared, and a likelihood ratio test confirmed that negative binomial was a better fit.

We first fit bivariate models for each of the independent variables (rainfall, LECZ, population density, and EC). This allowed us to explore district $\mathrm{PE} / \mathrm{E}$ rates as related to each predictor or independent variable of interest by itself. The predictor variables were defined as follows: LECZ categorized into 2 risk categories $(0-10 \mathrm{~m}$ high risk, $10+\mathrm{m}$ little/no risk), EC conductivity per $1000 \mu \mathrm{S} / \mathrm{cm}$, average rainfall per $100 \mathrm{~mm}$, and quartiles of population density (Cameron and Trivedi 2013). A final, fully adjusted model was then fit including all predictor variables. The full model also controlled for year of PE/E report, in case quality of data varied per year in the DHIS2 platform. 
District-level fixed effects were applied after conducting a Hausman test to compare model fit using random vs fixed effects. District-level fixed effects allow us to control for within-district variation over the time points and control for any unobservable factors that might affect the outcome. We considered but did not ultimately include any additional steps to control for spatial correlation, with such a small sample size $(n=$ 64 districts) and district-level fixed effect, our approach approximates a spatial regime model by stratifying the dataset and running the model by district.

Several sensitivity analyses were carried out, for example, running the models without the 2016 data (in case data quality had improved over time), using a binary dry vs rainy season instead of rainfall, and excluding Dhaka district because it is so densely urban. We also explored a binary variable for safe drinking water $(\leq 750 \mu \mathrm{S} / \mathrm{cm}$ or $>750 \mu \mathrm{S} / \mathrm{cm}$ ), the cut off defined by the WHO, instead of using EC conductivity per $1000 \mu \mathrm{S} / \mathrm{cm}$. A poverty variable was also explored but excluded due to high correlation with population density. These are not presented due to the similarity of the findings.

\section{Results}

Our dataset comprised 2304 observations for 36 months (2016-2018) across the 64 districts in Bangladesh. As expected, EC values are particularly high in coastal areas (with some overlap in the LECZ) (Fig. $1 \mathrm{a}$ and b). These coastal districts in the LECZ with higher EC values may experience increased salinity due to coastal intrusion from the sea or from practices such as saltwater shrimp farming and over-abstraction of groundwater for agriculture, which have been found to increase the salinity of surface and shallow groundwater water in nearby areas. There was strong correlation between elevation in meters and EC values reflected in the high risk LECZ category having much higher mean EC values $(7.43 \mu \mathrm{S} / \mathrm{cm})$ compared with the low risk LECZ category $(0.81 \mu \mathrm{S} / \mathrm{cm})($ Fig. 1c). Overall, the average district level EC value was $2254 \mu \mathrm{S} / \mathrm{cm}$ (min: $144 \mu \mathrm{S} / \mathrm{cm}$; max: 14,650 $\mu \mathrm{S} / \mathrm{cm}$ ); this resulted in 27 districts reporting mean values above the cut-off for safe drinking water (defined as $750 \mu \mathrm{S} / \mathrm{cm}$ ). However, these are district-level averages, and there may be significant variation within each district. A previous study testing water samples in coastal Bangladesh from different drinking water sources and neighboring households found significant spatial variability over small areas (Lam et al. 2018).

A map of the distribution of proportion of PE/E cases per district out of total facilitybased births in the rainy and dry season highlights some spatial variation (Fig. 2). Overall, the dry season has higher rates of $\mathrm{PE} / \mathrm{E}$, and the Barisal district on the coast reports the highest proportion of PE/E cases in both seasons. Some of the non-coastal districts with high rates are around Dhaka, which likely draws the most high-risk pregnancies and may have more consistent reporting. Also, the area in and around Dhaka likely has better medical services (potentially, more facility-based births, and more prompt diagnosis and treatment which may lead to fewer reports of $\mathrm{PE} / \mathrm{E}$ as a complication). On average, the proportion of pregnancies reported with $\mathrm{PE} / \mathrm{E}$ per month was $2.28(\mathrm{SD}=2.75)$ (Table 1$)$. This was higher in the dry season $(2.50(\mathrm{SD}=2.96)) \mathrm{vs}$ the rainy season $(2.06(\mathrm{SD}=2.50)$ ). Tabulating the proportion of monthly $\mathrm{PE} / \mathrm{E}$ cases and rainfall highlight their inverse relationship; the proportion of $\mathrm{PE} / \mathrm{E}$ cases is highest in the dry season (Fig. 3). The maximum rainfall was $700 \mathrm{~mm}$ in the 2017 rainy season, 
a

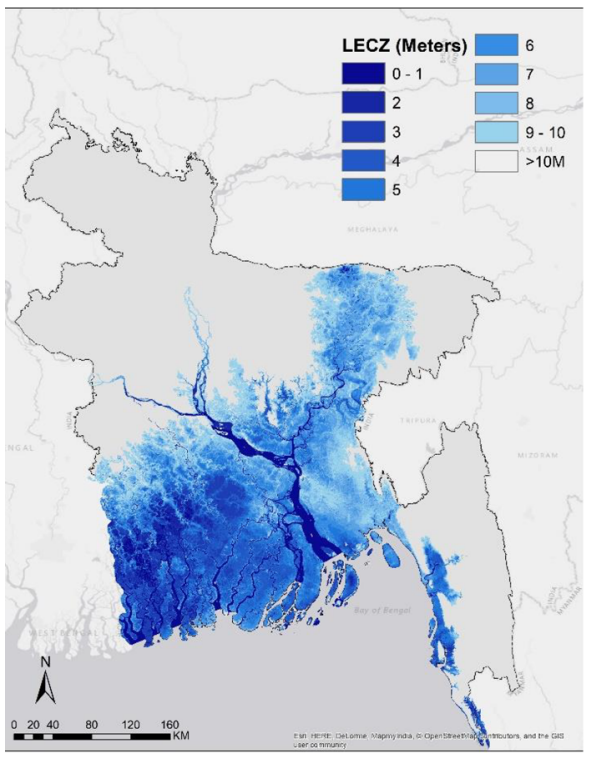

b

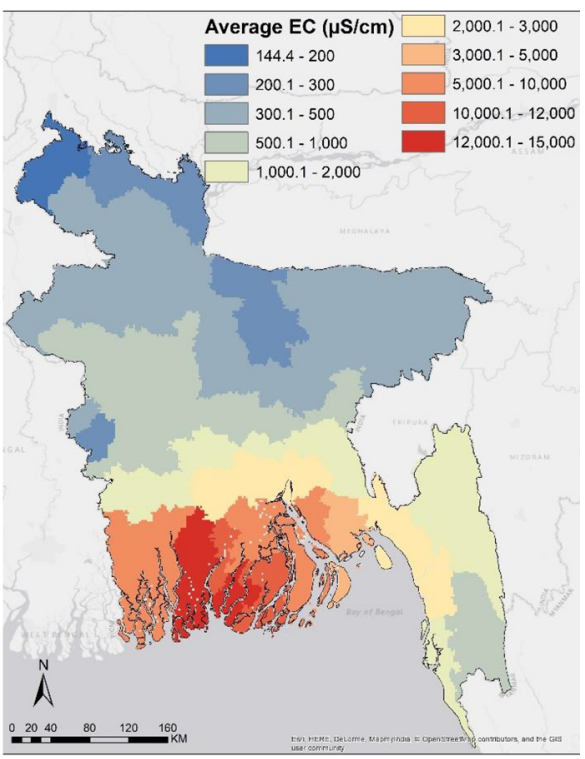

C

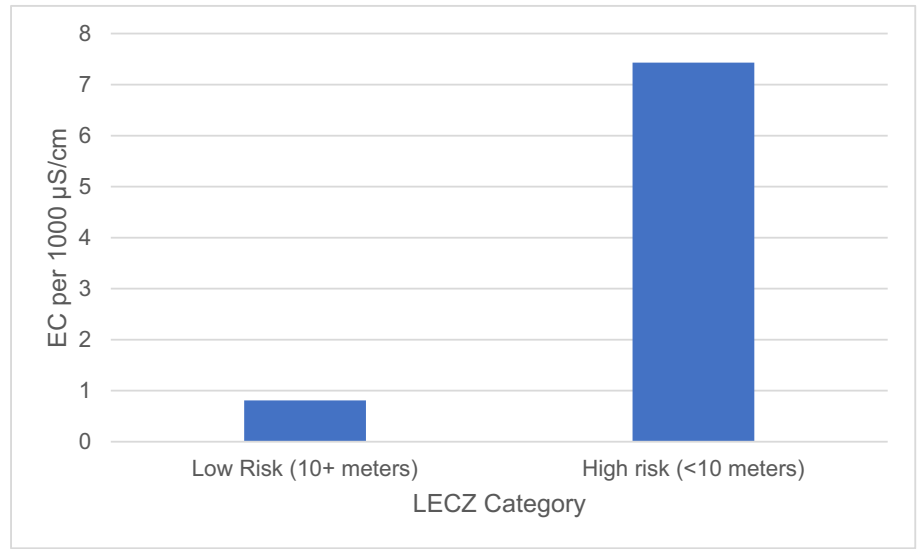

Fig. 1 Maps of environmental risks in Bangladesh. a Low elevation coastal zone (LECZ). b Electrical conductivity (EC)/salinity. c Average EC by LECZ risk category, Bangladesh

and minimum was $0 \mathrm{~mm}$ in each dry season. The proportion of $\mathrm{PE} / \mathrm{E}$ cases was also higher in the lowest population density districts and in districts with EC values $\geq 750$ $\mu / \mathrm{cm}$, which is considered the cut off for safe drinking water, and was highest in the high risk LECZ category of $0-10 \mathrm{~m}$ from the coast (Table 1).

Bivariate models were run first and are presented in Table 2, labeled as unadjusted model results. Next, a full negative binomial regression model with district level fixed effects was fit. The full model found that as EC increases $1000 \mu \mathrm{S} / \mathrm{cm}$, the risk of PE/E 


\section{a Rainy Season}

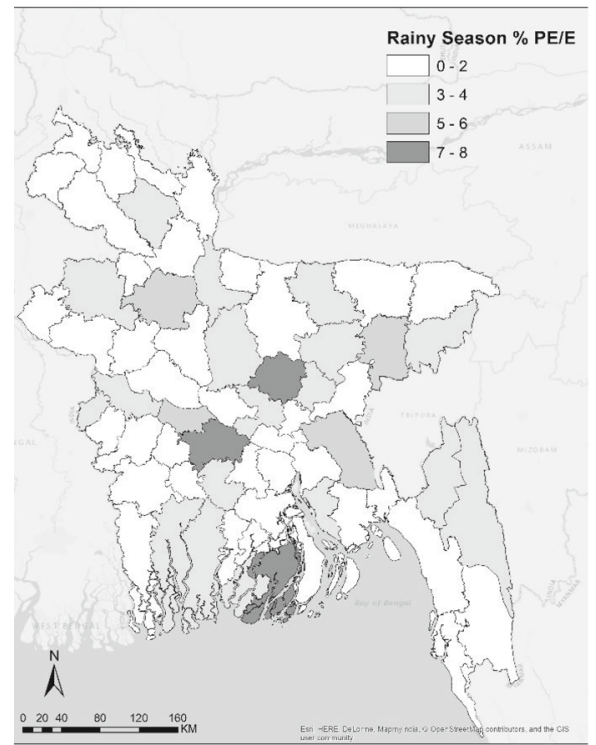

\section{b Dry Season}

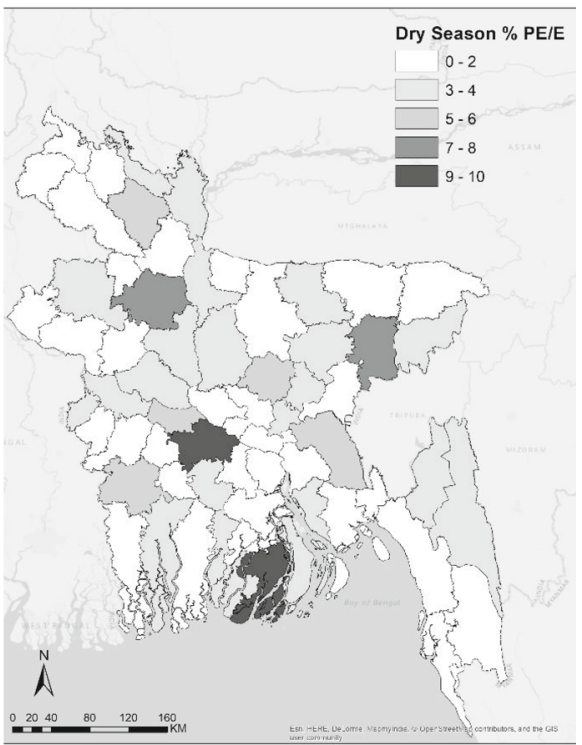

Fig. 2 District level reports of average PE/E cases out of total births reported Jan 2016-December 2018; by Season

increases 7\%, (IRR $=1.07,95 \%$ CI: 1.03, 1.11) (Table 2). As rainfall increases 100 $\mathrm{mm}$, the risk of PE/E decreases $4 \%$ (IRR $=0.96$; 95\% CI: 0.94, 0.97). This is consistent with previous findings that suggest in the dry season, due to evaporation and over-use of water sources, the salinity in surface and shallow groundwater increases. This

Table 1 Average PE/E rates by environmental factors in Bangladesh, 2016-2018

\begin{tabular}{lrr}
\hline & Mean & SD \\
\hline N observations & 2304 & \\
$\mathrm{~N}$ districts & 64 & \\
Proportion PE/E cases, overall & 2.28 & 2.75 \\
$\quad$ Year 2016 & 2.24 & 3.3 \\
$\quad$ Year 2017 & 2.26 & 2.43 \\
$\quad$ Year 2018 & 2.33 & 2.44 \\
Summary PE/E rates by environmental variables & & \\
Dry season & 2.50 & 2.96 \\
Rainy season & 2.06 & 2.50 \\
EC categories & & \\
Below safe water cut off $(\mathrm{EC}<750 \mu / \mathrm{cm})$ & 2.16 & 2.49 \\
Above safe water cut off $(\mathrm{EC} \geq 750 \mu / \mathrm{cm})$ & 2.43 & 3.07 \\
LECZ categories & & \\
$\quad 0-10 \mathrm{~m}$ & 2.37 & 2.68 \\
$\geq 10 \mathrm{~m}$ & 2.25 & 2.77 \\
\hline
\end{tabular}




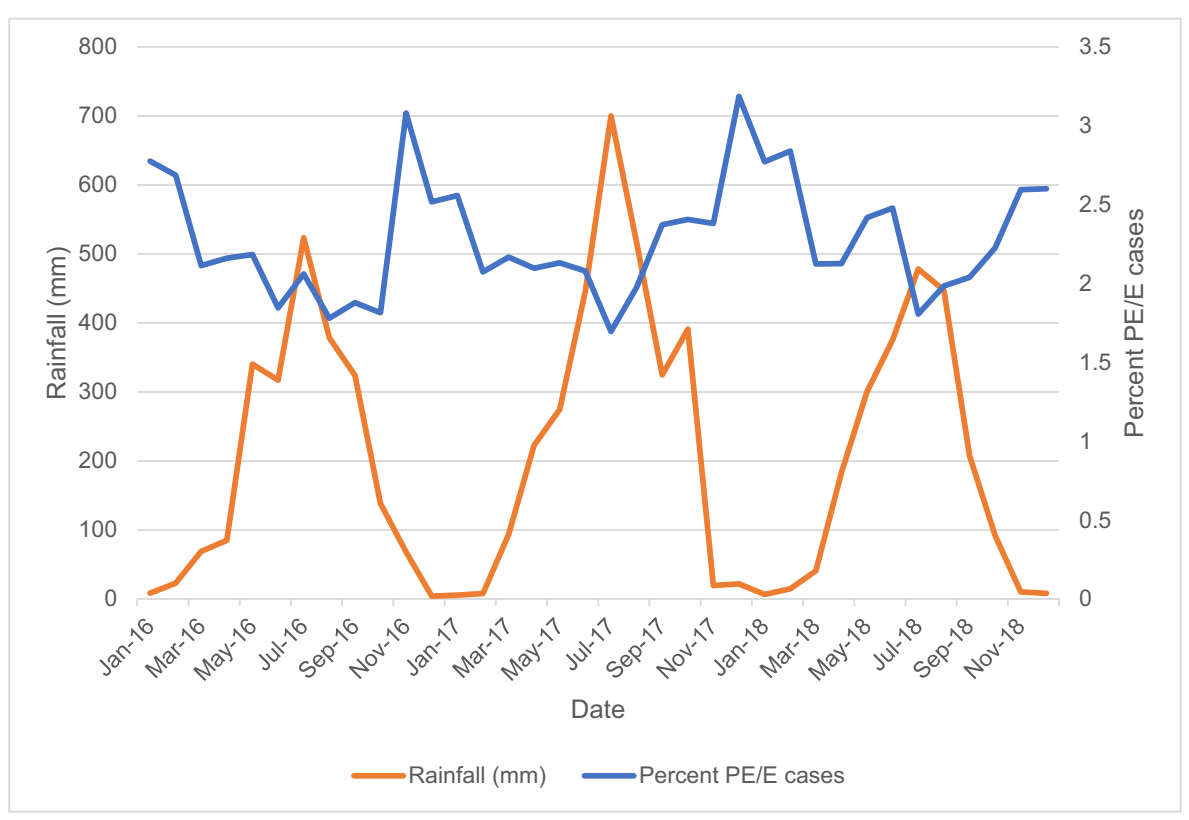

Fig. 3 Average monthly rainfall and total PE/E cases reported, 2016-2018

suggests that $\mathrm{EC}$ of groundwater is associated with $\mathrm{PE} / \mathrm{E}$ rates at the district level, with a seasonal pattern. Rates of $\mathrm{PE} / \mathrm{E}$ were also significantly lower in the high population density districts; comparing the most dense to the least dense, the risk of PE/E was $87 \%$ lower $(\mathrm{IRR}=0.13,95 \% \mathrm{CI}: 0.10,0.16)($ Table 2$)$. This suggests that districts with more rural livelihoods may rely more on natural water sources and also may have more limited access to health services (or lower health seeking behavior).

The relationship with LECZ is also detected. Compared with the low/no risk of coastal flooding category ( $>10 \mathrm{~m}$ from the coast), districts in the high risk category $(0-$ $10 \mathrm{~m}$ ) had a significantly higher risk of PE/E (IRR $=1.36 ; 95 \% \mathrm{CI}: 1.04,1.79)$ (Table 2). Although LECZ values are available at more granular levels, for this analysis, values were aggregated to the district level to match DHIS2. We explored smaller categories of elevation but due to the small sample size and high correlation between elevation and EC values, a simplified binary variable was selected. Future studies may disaggregate LECZ into finer categories if more granular data on PE/E become available.

\section{Discussion}

This study is the first to detect an association between district level rates of PE/E and drinking water salinity using national level health surveillance and environmental data. It also detects an inverse relationship between seasonally reported $\mathrm{PE} / \mathrm{E}$ rates by monthly rainfall. Overlaying health surveillance data with freely available remotely sensed data (such as rainfall and elevation) presents a low-cost methodology that can be applied to detect associations between environmental risk factors and health outcomes, 
Table 2 Negative binomial regression with district fixed effects of the association between PE/E cases and average district level environmental variables, Bangladesh 2016-2018

\begin{tabular}{|c|c|c|c|c|}
\hline & \multicolumn{2}{|c|}{ Unadjusted models } & \multicolumn{2}{|c|}{ Adjusted model } \\
\hline & IRR & $95 \%$ confidence interval & IRR & $95 \%$ confidence interval \\
\hline Avg EC (per $1000 \mu \mathrm{S} / \mathrm{cm}$ ) & $1.19 * * *$ & $1.16,1.21$ & $1.07 * *$ & $1.03,1.11$ \\
\hline \multicolumn{5}{|l|}{ LECZ } \\
\hline Low/no risk $(10+m)$ & REF & & REF & \\
\hline High risk $(<10 \mathrm{~m})$ & $2.59 * * *$ & $2.19,3.06$ & $1.36^{*}$ & $1.04,1.79$ \\
\hline \multicolumn{5}{|l|}{ Population density } \\
\hline Quartile 1 (least dense) & REF & & REF & \\
\hline Quartile 2 & $0.61 * * *$ & $0.49,0.76$ & $0.72 * *$ & $0.57,0.91$ \\
\hline Quartile 3 & $0.31 * * *$ & $0.25,0.38$ & $0.40 * * *$ & $0.32,0.49$ \\
\hline Quartile 4 (most dense) & $0.10^{* * *}$ & $0.08,0.12$ & $0.13 * * *$ & $0.10,0.16$ \\
\hline Rainfall (per 100 mm) & $0.97 * * *$ & $0.96,0.98$ & $0.96 * * *$ & $0.94,0.97$ \\
\hline \multicolumn{5}{|l|}{ Year } \\
\hline 2016 & REF & & REF & \\
\hline 2017 & $1.26^{* * *}$ & $1.17,1.35$ & $1.14 * * *$ & $1.06,1.21$ \\
\hline 2018 & $1.22 * * *$ & $1.13,1.31$ & $1.14 * * *$ & $1.07,1.21$ \\
\hline
\end{tabular}

$* p \leq 0.05$

$* * p \leq 0.01$

$* * * p \leq 0.001$

identify and predict hotspots, and inform targeting of public health programs. Surveillance data also allows for routine monitoring of health outcomes such as PE/E, to measure progress and achievement against benchmarks. This will be increasingly important as the negative health effects related to climate change and sea level rise affect coastal regions all over the world. Projections suggest global sea levels will rise 1 m or more by 2100 , putting many coastal communities at risk, particularly countries in South, Southeast, and East Asia because of their densely populated delta regions (Nicholls and Cazenave 2010). An estimated 25 million people across these regions are at risk of drinking saline water (Shammi et al. 2019).

Salinity in surface and groundwater is determined by environmental features, anthropogenic changes, and land management practices, as well as by sea level rise and other climate related factors (Khan et al. 2011). With rising sea levels, salinity intrusion will extend further and further inland making coastal regions with low elevation, such as Bangladesh, particularly vulnerable. Already, initial studies show that populations in coastal Bangladesh are drinking unacceptably high salinity water. While the current WHO guidelines are $2 \mathrm{~g}$ /day of dietary sodium intake from all sources, in Dacope, the estimates are 5-16 g/day in drinking water alone during the dry season (Khan et al. 2011). Bangladesh is not the only place where salinity is an issue, but so far, it is the focus of research into the effects of drinking water salinity on health. As concerns regarding drinking water salinity increase, studies should be conducted in other coastal regions and countries, and future research will be necessary 
to understand how these communities can adapt and access alternative sources of safe drinking water.

In Bangladesh, as in many low-income countries, most of the rural population accesses drinking water from tube wells. Consuming sodium has been linked with immunological effects on skin tissue, intestinal microbiology, and other organs, as well as cardiovascular disease, inflammation, infection, and autoimmunity (Shammi et al. 2019); it has also been associated with hypertension, infant mortality, and PE/E (Khan et al. 2011, Khan et al. 2014, Talukder et al. 2016, Nahian et al. 2018). When groundwater is too saline, people report harvesting rainwater to drink instead. However, drinking rainwater may pose other health risks, as rainwater may be contaminated with dust or water-borne disease pathogens, and its storage may create breeding sites for mosquitoes that carry vector-borne diseases such as malaria, dengue, and chikungunya (Hoque et al. 2016). Other interventions being explored to address salinity include pond sand filter systems, managed aquifer recharge (MAR), solar-powered desalination plants, and reverse osmosis methods (Shammi et al. 2019). Another potential area for exploration for blood pressure, specifically, is described in a recent study that found water with higher levels of calcium and magnesium were linked with lower blood pressure even if sodium levels were high (Naser et al. 2019. Low cost, accessible, and dependable safe drinking water solutions must be evaluated and deployed in the region.

Our study also confirmed a seasonal pattern between PE/E and rainfall, suggested in an earlier study. The seasonal link is interesting but the relationship is not clear; PE/E has been shown to follow a seasonal pattern in sub-Saharan Africa and Central and South Asia, but the mechanism is not well understood (Hlimi 2015). A systematic review found in some settings that $\mathrm{PE} / \mathrm{E}$ was higher in the rainy season, potentially due to malaria infection, while other studies have found higher rates in the dry season, potentially due to nutritional deficiencies linked to a shortage of food in this time (Hlimi 2015). In Bangladesh, we hypothesize that the seasonal variation in drinking water salinity may be linked with the seasonal rates of $\mathrm{PE} / \mathrm{E}$, due to women in coastal regions drinking groundwater that becomes more saline when there is less rainfall. Khan et al. posit that the seasonal variation may be because coastal communities cannot obtain sufficient drinking water from rainwater harvesting during the winter and must therefore use groundwater and ponds as drinking water sources during these months (Khan et al. 2011). Additional research is necessary to understand the mechanism and timing between saline water exposure and pregnancy outcomes; for example, it is unclear if drinking saline water during a specific trimester of pregnancy is more likely to lead to adverse outcomes.

One strategy to monitor environment and health patterns is to use surveillance data and remotely sensed/satellite derived datasets that can be linked by spatial location, as presented in this analysis. Linking rainfall with platforms such as DHIS2 may yield useful information for spatial and temporal targeting of interventions; this type of analysis has been done for other diseases such as malaria that have a seasonal relationship (Gunda et al. 2017). Despite the limitations of surveillance data, there are many opportunities to use it to detect these larger spatial-temporal relationships. The previous studies assessing salinity and PE/E have had to conduct intensive data collection, not feasible at the national level. Surveillance data is low cost, and in Bangladesh, reporting is required of all public health facilities. Work is ongoing to improve the accuracy and completeness of health surveillance data in developing 
countries and to address potential sources of bias in the data, but it's utility and application should continue to be explored.

This analysis has several limitations. First, DHIS2 data at the facility and upazila (sub-district administrative units) level are being collected but are not complete in the DHIS2 platform, so it was not possible to use them for this analysis. District level data was considered complete, but the small number of districts limited our analysis and the level of granularity we could explore. Second, the DHIS2 data are subject to surveillance data quality issues and biases. For example, DHIS2 does not account for births at home, referrals to other districts, and can only include PE/E cases that are reported to facilities. There may be more cases of $\mathrm{PE} / \mathrm{E}$ that go undetected due to underreporting or are missing altogether in DHIS2; for example, many facilities in Dhaka are private and excluded from the platform. Facilities with broken blood pressure monitors cannot report PE/E. Conversely, surveillance rates could be higher because high-risk pregnancies are referred to the facility and more likely to be reported. If these coastal facilities are more aware of $\mathrm{PE} / \mathrm{E}$ and more likely to test and report cases, this may bias the data; alternatively, because of this heightened awareness, they may identify and prevent more cases. A third limitation is that the environmental exposure variables had to be aggregated to the district level, even though it is available at much higher resolution. A previous study testing water samples in coastal Bangladesh from different drinking water sources and neighboring households found significant spatial variability over small areas (Lam et al. 2018). We still detected an association at the district level, but there may be an opportunity in the future to explore finer levels of data (for example, linking health facility GPS points or households to elevation and rainfall grid cell values). Future analyses can overlay health facility GPS points or the 492 upazilas for a much more granular analysis.

Pre-eclampsia, if detected early, can be monitored and controlled with antihypertensive drugs recommended by $\mathrm{WHO}$ for use during pregnancy, and $\mathrm{MgSO}_{4}$ to manage severe pre-eclampsia and control eclamptic seizures (WHO 2011). Early detection, monitoring, and management are critical to protecting pregnant and postnatal women and their newborns from the effects of $\mathrm{PE} / \mathrm{E}$. To reduce complications and death due to hypertensive disorders in pregnancy in Bangladesh, increased exposure to saline drinking water may be an important risk factor. Climate change, coastal flooding, shrimp farming, and agricultural practices are rendering coastal waters around the world increasingly saline, creating a need for alternative sources of safe drinking water. Public health surveillance data is a low cost tool that can be leveraged to explore the relationship between salinity and $\mathrm{PE} / \mathrm{E}$ in Bangladesh and should be expanded to other countries and settings. The results can inform the targeting of interventions to improve safe drinking water (such as managed aquifer recharge systems and desalination plants). In the future, health systems may routinely integrate available health data with environmental and climate data to detect and ultimately predict hotspots. Interventions could then be targeted, potentially in real-time, to these locations, streamlining health service delivery and maximizing the impacts on health in resource constrained settings. Future research should incorporate measures of salinity over time (in addition to rainfall) to more closely measure the interactions between salinity, rainfall, $\mathrm{PE} / \mathrm{E}$, and potentially the timing of exposure throughout a pregnancy. The methods and results presented here can be applied more broadly to all coastal regions that may experience saline intrusion and its negative health effects. 
Open Access This article is distributed under the terms of the Creative Commons Attribution 4.0 International License (http://creativecommons.org/licenses/by/4.0/), which permits unrestricted use, distribution, and reproduction in any medium, provided you give appropriate credit to the original author(s) and the source, provide a link to the Creative Commons license, and indicate if changes were made.

\section{References}

ACOG (2013). Magnesium sulfate use in obstetrics: committee opinion \#652, American College of Obstetricians and Gynecologists: e52-53.

Assembly, U. G. (2011). Political declaration of the high-level meeting of the General Assembly on the prevention and control of non-communicable diseases. United Nations: New York.

Cameron, A. and P. Trivedi (2013). Regression analysis of count data. Econometric Society Monographs. S. B. A Prat, Cambridge University Press.

Dasgupta, S., M. Huq and D. Wheeler (2015). Policy research working paper 7200: drinking water salinity and infant mortality in coastal Bangladesh, Environment and Energy Team, Development Research Group; World Bank.

Dehnavieh, R., Haghdoost, A., Khosravi, A., Hoseinabadi, F., Rahimi, H., Poursheikhali, A., Khajehpour, N., Khajeh, Z., Mirshekari, N., Hasani, M., Radmerikhi, S., Haghighi, H., Mehrolhassani, M., Kazemi, E., \& Aghamohamadi, S. (2019). The District Health Information System (DHIS2): a literature review and meta-synthesis of its strengths and operational challenges based on the experiences of 11 countries. Health Information Management Journal, 48(2), 62-75.

Duckitt, K. and D. Harrington (2005). "Risk factors for pre-eclampsia at antenatal booking: a systematic review of controlled studies.” BMJ 330.

Duley, L., D. Henderson-Smart, and S. Meher (2005). “Altered dietary salt for preventing pre-eclampsia, and its complications." Cochrane Database of Systematic Reviews 4.

Elmugabil, A., H. Hamdan, A. Elsheikh, D. Rayis, I. Adam and G. Gasim (2016). "Serum calcium, magnesium, zinc and copper levels in Sudanese women with preeclampsia." PLoS ONE 11(12).

EPA. (2014). Drinking water advisory: Consumer acceptability advice and health effects analysis on sodium. Washington: Environmental Protection Agency.

Funk, C., P. Peterson, M. Landsfeld, D. Pedreros, J. Verdin, S. Shukla, G. Husak, J. Rowland, L. Harrison, A. Hoell, and J. Michaelsen (2015). "The climate hazards infrared precipitation with stations - a new environmental record for monitoring extremes." Scientific Data 2.

Gunda, R., M. Chimbari, S. Shamu, B. Sartorius and S. Mukaratirwa (2017). "Malaria incidence trends and their assocation with climatic variables in rural Gwanda, Zimbabwe, 2005-2015." Malaria Journal 16(393).

Hasan, M., Shamsuddin, M., \& Hossain, A. (2013). Salinity status in groundwater: a study of selected Upazilas of southwestern coastal region in Bangladesh. Gloal Science and Technology Journal, 1(1), $112-122$.

Haugen, J., G. Hjemas and O. Poppe (2017). "Manual for the DHIS2 quality tool: understanding the basics of improving data quality." Statistics Norway.

Hawker, L., P. Bates, J. NEal and J. Rougier (2018). "Perspectives on digital elevation model (DEM) simulation for flood modeling in the absence of a high-accuracy open access global DEM." Frontiers in Earth Science.

He, F., \& Macgregor, G. (2009). A comprehensive review on salt and health and current experience of worldwide salt reduction programmes. Journal of Human Hypertension, 23(6), 363-384.

Hlimi, T. (2015). Association of anemia, pre-eclampsia, and eclampsia with seasonality: a realist systematic review. Health \& Place, 31, 180-192.

Hoque, M., Scheelbeek, P., Vineis, P., Khan, A., Ahmed, K., \& Butler, A. (2016). Drinking water vulnerability to climate change and alternatives for adaptation in coastal South and South East Asia. Climatic Change, 136, 247-263.

Khan, A., S. Mojumder, S. Kovats and P. Vineis (2008). "Saline contamination of drinking water in Bangladesh." Lancet 371.

Khan, A., A. Ireson, S. Kovats, S. Mojumder, A. Khusru, A. Rahman and P. Vineis (2011). "Drinking water salinity and maternal health in coastal Bangladesh: implications of climate change." Environmental Health Perspectives 119(9). 
Khan, A., P. Scheelbeek, A. Shilpi, Q. Chan, S. Mojumder, A. Rahman, A. Haines and P. Vineis (2014). "Salinity in drinking water and the risk of (pre)eclampsia and gestational hypertension in coastal Bangladesh: a case-control study." PLoS ONE 9(9).

Kirk, K., \& Dempsey, A. (2016). A systematic review of the treatment and management of pre-eclampsia and eclampsia in Bangladesh. Population Council: Washington.

Lam, Y., P. Surkan, P. Winch and F. Nizame (2018). "Freshwater access in high salinity regions: impacts and adaptation insights from the Ganges River Delta." Journal of Global Health Reports 2.

Lapworth, D., A. Zahid, R. Taylor, W. Burgess, M. Shamsudduha, K. Ahmed, A. Mukherjee, D. Gooddy, D. Chatterjee, and A. MacDonald (2018). "Security of deep groundater in the coastal Bengal Basin revealed by tracers." Geophysical Research Letters 45.

Lichter, M., Vafeidis, A., Nicholls, R., \& Kaiser, G. (2011). Exploring data-related uncertainties in analyses of land area and population in the 'low-elevation coastal zone. Journal of Coastal Research, 27, 757-768.

MacManus, K., Balk, D., Engin, H., \& McGranahan, G. (2019). Modeling the impacts of sea level rise globally: low elevation coastal zones based on new sources. San Francisco: American Geophysical Union.

Magee, L., Pels, A., Helewa, M., Rey, E., Dadelszen, P. V., \& C. H. D. O. P. H. W. Group. (2016). The hypertensive disorders of pregnancy. Best Practice \& Research in Clinical Obstetrics \& Gynaecology, 29, 643-657.

McGranahan, G., Balk, D., \& Anderson, B. (2007). The rising tide: assessing the risks of climate change and human settlements in low elevation coastal zones. Environment and Urbanization, 19, 17-37.

McMichael, A. (2003). Climate change and human health: risks and responses. Geneva: World Health Organization.

MOEF. (2006). Impact of sea-level rise on land use suitability and adaptation options: coastal land zoning in the Southwest. Ministry of Environment and Forest: Dhaka.

Mone, F. and F. MCAuliffe (2014). "Low-dose aspirin and calcium supplementation for the prevention of preeclampsia." Obstetrics \& Gynaecology.

Nahian, M., A. Ahmed, A. Lazar, C. Hutton, M. Salehin and P. Streatfield (2018). "Drinking water salinity associated health crisis in coastal Bangladesh.” Elementa: Science of the Anthropocene 6(2).

Naser, A., M. Rahman, L. Unicomb, S. Doza, M. Gazi, G. Alam, M. Karim, M. Uddin, G. Khan, K. Ahmen, M. Shamsudduha, S. anad, K. Narayan, H. Chang, S. Luby, M. Gribble and T. Clasen (2019). "Drinking water salinity, urinary macro-mineral excretions, and blood pressure in the southwest coastal population of Bangladesh." Journal of the American Heart Assocation.

National Institute of Population Research and Training (NIPORT), I. C. f. D. D. R., Bangladesh (icddr,b), and MEASURE Evaluation (2017). Bangladesh maternal mortality and health care survey 2016: preliminary report. Dhaka, Bangladesh and Chapel Hill, NC, USA, NIPORT, icddr,b, and MEASURE Evaluation.

Nicholls, R. and A. Cazenave (2010). "Sea-level rise and its impact on coastal zones." Science 328.

Redman, C. (2011). Hypertension in pregnancy: the NICE guidelines. Heart, 97(23), 1967-1969.

Say, L., Chou, D., Gemmill, A., Tuncalp, O., Moller, A.-B., Daniels, J., Gulmezoglu, A., Temmerman, M., \& Alkema, L. (2014). Global cause of maternal death: a WHO systematic analysis. Lancet Global Health, 2(6), PE323-PE333.

Scheelbeek, P., M. Chowdhury, A. Haines, D. Alam, M. Hoque, A. Butler, A. Khan, S. Mojunder, M. Blangiardo, P. Elliott and P. Vineis (2016). "Drinking water salinitiy and raised blood pressure: evidence from a cohort study in coastal Bangladesh." Environmental Health Perspectives.

Shammi, M., M. Rahman, M. Islam, M. Bodrud-Doza, A. Zahid, Y. Akter, S. Quaiyum and M. Kurasaki (2017). "Spatio-temporal assessment and trend analysis of surface water salinity in the coastal region of Bangladesh." Environmental Science and Pollution Research.

Shammi, M., M. Rahman, S. Bondad and M. Bodrud-Doza (2019). "Impacts of salinity intrusion in community health: a review of experiences on drinking water sodium from coastal areas of Bangladesh." Healthcare 7(50).

Shamsudduha, M., G. Joseph, S. Haque, M. Khan, A. Zahid and K. Ahmed (2019). Multi-hazard groundwater risks to the drinking water supply in Bangladesh: challenges to achieving the sustainable development goals. Policy Research Working Paper, World Bank, Washington DC.

Shamsudduha, M., G. Joseph, S. Haque, M. Khan, A. Zahid, K. Ahmed (2019). Multi-hazard Groundwater Risks to Water Supply from Shallow Depths: Challenges to Achieving the Sustainable Development Goals in Bangladesh. Exposure \& Health. https://doi.org/10.1007 /s12403-019-00325-9 
Talukder, M., Rutherford, S., Phung, D., Islam, M., \& Chu, C. (2016). The effect of drinking water salinity on blood pressure in young adults of coastal Bangladesh. Environmental Pollution, 214, $248-254$

WHO. (2011). "WHO recommendations for prevention and treatment pre-eclampsia and eclampisa." Retrieved 5/16, 2019, from https://apps.who.int/iris/bitstream/handle/10665/44703/9789241548335_ eng.pdf.

Xu, M., Guo, D., Gu, H., Zhang, L., \& Lv, S. (2016). Selenium and preeclampsia: a systematic review and meta-analysis. Biological Trace Element Research, 171(2), 283-292.

Yamazaki, D., Ikeshima, D., Tawatari, R., Yamaguchi, T., O’Loughlin, F., Neal, J., Sampson, C., Kanae, S., \& Bates, P. (2017). A high accuracy map of global terrain elevations. Geophysical Research Letters, 44, 5844-5853.

Zahid, A., Jahan, K., Ali, M., Ahmed, N., Islam, M., \& Rahman, A. (2013). Distribution of groundwater salinity and its seasonal variability in the coastal aquifers of Bengal Delta (pp. 170-193). Bangladesh: Impact of Climate Change on Socio-economic Conditions of Bangladesh. G. A. E. S. D. Alumni Association of German Universities in Bangladesh. Dhaka.

Publisher's note Springer Nature remains neutral with regard to jurisdictional claims in published maps and institutional affiliations. 